\title{
AN ADDENDUM TO THE GENERAL COEFFICIENT THEOREM
}

BY

\author{
JAMES A. JENKINS(1)
}

1. In our ultimate form of the General Coefficient Theorem [1, Theorem 1] we imposed a normalization on the expression for the quadratic differential at a pole of order greater than two. The purpose of this was to avoid complication in the calculations given in [1, pp. 393-394]. It was observed that this normalization could always be attained by a suitable change in the local parameter employed at the pole in question [1, Remark, p. 389]. The object of the present paper is to show that using the form of the General Coefficient Theorem obtained there we can without essential difficulty obtain the appropriate formulation when we drop the normalization in question. We will use throughout the notation of [1].

2. The result we prove has the following form.

Addendum to the General Coefficient Theorem. Let the pole $P_{j}$ of $Q(z) d z^{2}$ of order $m_{j}$ greater than two lie in the domain $\Delta_{l}$ and in terms of a local parameter $z$ representing $P_{j}$ as the point at infinity let $f_{l}$ have the expansion

and $Q$ the expansion

$$
f_{l}(z)=z+\sum_{i=k}^{\infty} \frac{a_{i}^{(j)}}{z^{i}}
$$

$$
Q(z)=\alpha^{(j)}\left[z^{m_{j}-4}+\sum_{i=1}^{\infty} \beta_{i}^{(j)} z^{m_{j}-i-4}\right] .
$$

Then the contribution from the pole $P_{j}$ to the expression whose real part constitutes the left-hand side of the fundamental inequality in the General Coefficient Theorem $[1,(6)]$ is

if $m_{j}$ is odd and

$$
\alpha^{(j)}\left[a_{m_{-}-3}^{(j)}+\sum_{i=1}^{k_{j}} \beta_{i}^{(j)} a_{m_{-}-i-3}^{(j)}\right],
$$

$$
\alpha^{(j)}\left[a_{m_{J}-3}^{(j)}+\sum_{i=1}^{k_{j}+1} \beta_{i}^{(j)} a_{m_{J}-i-3}^{(j)}+\frac{1}{2}\left(\frac{1}{2} m_{j}-2\right)\left(a_{k_{f}}^{(j)}\right)^{2}\right]
$$

if $m_{j}$ is even.

Received by the editors March 30, 1962.

(1) Supported by the Air Force Office of Scientific Research. 
The essential step is to prove the following lemma.

LEMMA. The appropriate expression (1) or (2) (according as $m_{j}$ is odd or even) is invariant under change of local parameter at $P_{j}$ (always with the assumption that $P_{j}$ be represented by the point at infinity).

From now on to simplify notation we will omit all subscripts and superscripts $j$ and $l$.

It is evident that any change of local parameter can be broken down into a finite succession of changes of the form:

$$
\begin{array}{ll}
z=\kappa \tilde{z}, & \\
z=\tilde{z}+\frac{c_{r}}{\tilde{z}^{r}}, & r=0,1, \cdots, R, \\
z=\tilde{z}+\sum_{j=R+1}^{\infty} \frac{c_{j}}{\tilde{z}^{j}} . &
\end{array}
$$

Under a change of parameter of type (5) for $R$ large enough it is clear that each coefficient appearing in (1) or (2) is invariant; thus the result of the lemma is clear in this case.

For a change of parameter of type (3) we have the new expressions for the quadratic differential and the mapping:

so that

$$
\begin{aligned}
\tilde{Q}(\tilde{z}) & =\tilde{\alpha}\left[\tilde{z}^{m-4}+\sum_{i=1}^{\infty} \tilde{\beta}_{i} \tilde{z}^{m-i-4}\right] \\
& =\alpha\left[\kappa^{m-4} \tilde{z}^{m-4}+\sum_{i=1}^{\infty} \beta_{i} \kappa^{m-i-4} \tilde{z}^{m-i-4}\right] \kappa^{2},
\end{aligned}
$$

and

$$
\tilde{\alpha}=\kappa^{m-2} \alpha, \tilde{\beta}_{i}=\kappa^{-i} \beta_{i}
$$

$$
\tilde{f}(\tilde{z})=\tilde{z}+\sum_{i=k}^{\infty} \frac{\tilde{a}_{i}}{\tilde{z}^{i}}
$$

where

$$
\kappa \tilde{f}(\tilde{z})=\kappa \tilde{z}+\sum_{i=k}^{\infty} \frac{\kappa^{-i} a_{i}}{\tilde{z}^{i}}
$$

or

so that

$$
\tilde{f}(\tilde{z})=\tilde{z}+\sum_{i=k}^{\infty} \frac{\kappa^{-(i+1)} a_{i}}{\tilde{z}^{i}}
$$

$$
\tilde{a}_{i}=\kappa^{-(i+1)} a_{i}
$$

It is verified at once that the appropriate expression (1) or (2) is invariant. 
Finally for a change of parameter of type (4) we have for the mapping the expression

where

$$
\tilde{f}(\tilde{z})=\tilde{z}+\sum_{i=k}^{\infty} \frac{\tilde{a}_{i}}{\tilde{z}^{i}}
$$

$$
\tilde{f}(\tilde{z})+c_{r}(\tilde{f}(\tilde{z}))^{-r}=\tilde{z}+c_{r} \tilde{z}^{-r}+\sum_{i=k}^{\infty} a_{i}\left(\tilde{z}+c_{r} \tilde{z}^{-r}\right)^{-i} .
$$

The left-hand side of (3) is equal to

$$
\tilde{z}+\sum_{i=k}^{\infty} \tilde{a}_{i} \tilde{z}^{-i}+c_{r} \tilde{z}^{-r}\left(1+\sum_{i=k}^{\infty} \tilde{a}_{i} \tilde{z}^{-(i+1)}\right)^{-r},
$$

that is,

$$
\begin{aligned}
\tilde{z}+c_{r} \tilde{z}^{-r} & +\sum_{i=k}^{k+r} \tilde{a}_{i} \tilde{z}^{-i}+\sum_{i=k+r+1}^{2 k+r+1}\left(\tilde{a}_{i}-r c_{r} \tilde{a}_{i-r-1}\right) \tilde{z}^{-i} \\
& + \text { higher powers of } \tilde{z}^{-1} .
\end{aligned}
$$

Thus insofar as its coefficients of $z^{-k}, \cdots, z^{-(m-3)}$ are concerned

$$
\left(\sum_{i=k}^{m-3} a_{i} z^{-i}\right)(d z)^{-1}
$$

transforms as though it were a reciprocal differential. Thus the product

$$
\left(\sum_{i=k}^{m-3} a_{i} z^{-i}\right) Q(z) d z
$$

insofar as terms involving only these coefficients are concerned transforms as though it were a linear differential. In particular the residue at the pole is such a term and is thus an invariant. However, the residue has the value

$$
-\alpha\left[a_{m-3}+\sum_{i=1}^{k} \beta_{i} a_{m-i-3}\right]
$$

if $m$ is odd and

$$
-\alpha\left[a_{m-3}+\sum_{i=1}^{k+1} \beta_{i} a_{m-i-3}\right]
$$

if $m$ is even.

To complete the proof of the lemma we need only observe that in the case of $m$ even the terms $\alpha$ and $a_{k}$ are each invariant under a change of local parameter of type (4). 
Our principal result follows at once from the lemma on observing that if we have $\beta_{i}=0, i=1, \cdots, k$, the ter $\mathrm{m}$ (1) or (2) reduces to the expression appearing in $[1$, Theorem 1].

\section{BIBLIOGRAPHY}

1. James A. Jenkins, An extension of the General Coefficient Theorem, Trans. Amer. Math. Soc. 95 (1960), 387-407.

WASHINGTON UNIVERSTTY,

ST. Louns, MissourI 